Studia UBB 㱠igitalia, Volume 62 (LXII) 2017, June, Issue 1, 112-132

Published Online: 2017-06-30

DOI:10.24193/subbdigitalia.2017.1.09

\title{
The digitisation - measuring physical and virtual presence
}

\author{
Liana Stanca*, Cristina Felea, \\ Ramona Lacurezeanu, Christian Schuster \\ Babeș-Bolyai University, Cluj-Napoca, Romania, \\ *Corresponding author: liana.stanca@econ.ubbcluj.ro
}

\begin{abstract}
The aim of the present study is to create the profile of persons who have become aware of their need to have a specific tool to move intelligently in an urban space. The research is conducted within the frame of smart cities literature and approaches cities from the perspective of the presence of Information and Communication Technologies in all areas of social, economic and political life. In this context, smart people are considered to be persons who use the advantages of technology to obtain information-knowledge and are capable to identify the need of a smart tool to assist them in various domains. A concept map is created that is interpreted as a tool to measure the physical and virtual presence of senior undergraduate students in Economic Informatics in places significant for academic information and knowledge transfer. The authors try to demonstrate the importance of combining data mining and social network analysis methods so as to carry out a complex analysis of the problem and validate by their findings important research by Fortunato, Girvan, Papadopoulos, Adedoyin-Olowe et al.
\end{abstract}

Keywords: smart people; behaviour pattern; concept map; knowledge; information flow; social network analysis; data mining.

\section{Introduction}

In the past decade, urban development has undergone major transformations due to the presence of information and communication technologies (ICT-s). The impact of technology on various aspects of urban development and the resulting new structures and relationships have been subject to a labelling process that attach to city 
determinants such as 'informational', 'intelligent', 'hi-tech', 'tele-', 'online', 'digital', 'smart', etc. In his ground-breaking 2008 article, Hollands approaches critically the phenomenon of what he calls 'urban labelling' stating that there is no scientifically founded definition of 'smart' cities, due to the complex variables that enter discussion. The transition from 'intelligent' to 'smart' cities, he claims, should be based on more than information and communication technologies used. As a conclusion, he offers his own provisional definition whereby "smart cities can be understood as a high-tech variation of the "entrepreneurial city"' and speculates on some general principles that would make smart cities more "progressive and inclusive". Various definitions of the concept have been proposed. For instance, Kominos, Ionita and llie, Paskaleva mention the concepts of "Telecities", "Cities On Line", "Intelligent cities", "Digital cities" that are used in the European Union for projects and local programs that stimulate the development of information technology at the level of online cities.

The European Smart Cities Project (started in 2007) provides rich data from a comparative analysis of $70+$ cities' economic, social and geographical features to provide a hierarchy of the best places to conduct particular activities. Researchers proposed six major dimensions for generating a consistent ranking: Smart Economy, Smart People, Smart Governance, Smart Mobility, Smart Environment, and Smart Living (Giffinger et al.) focused on medium-sized cities, in an attempt to prove that these are engaged in efforts to become smart in order to compete with large metropolises. Within the study, Luxembourg is ranked first in the general hierarchy and Timisoara, Romania, is ranked 12 in the Smart People category, with position 51 in the general classification, labelled as smart city (medium smart in terms of smart mobility and people). In their paper, (Androniceanu, Ivan) drew on this project (Giffinger et al.) and tried to add more criteria needed in the further analysis of smart cities, such as transparency, responsibility, flexibility, durability, competitiveness, and benchmarking. The authors also mention that, if analysed according to the grid of Giffinger et al., Cluj-Napoca, Romania meets the conditions for a smart city, namely the number of inhabitants, public and private universities, a dynamic economy, and an IT and software centre. In their turn, Kominos, Shapiro, and Soom all consider that the six dimensions mentioned in the European Smart Cities Project report are a result/continuation of traditional regional and neoclassical urban and economic development theories such as regional competitiveness, transport and ICT economy, natural resources, human and social capital, quality of life and civic participation to city management. Lombardi considers smart cities to include participation, security/safety, and cultural heritage. Consequently, in all mentioned studies, the following major characteristics or clusters of features are mentioned: smart governance (participation), smart human capital (smart people), smart environment (natural resources), smart life conditions (quality of life), smart economy (competitiveness). In the call for papers for the 2015 Media City $5^{\text {th }}$ International Conference and Exhibition (Plymouth University, 
UK) dedicated to urban cultural studies, the organisers propose two major paradigms for approaching virtual cities. On the one hand, emphasis is put on a "data centred" change of the urban space in relation to technology, with focus on the optimisation of urban infrastructure/systems/technology to the benefit of citizens. In this view, the city should change and offer people customised/personalised services. Urban ICT-s are expected to generate new spatial-temporal participative patterns and will diminish people's participation, gradually eliminating their physical presence in favour of the virtual, which will be measured by "likes". On the other hand, a "citizen driven" approach is proposed, with unplanned, informal practices generating "counter-cultural" scenarios in which citizens are increasingly present in the transformations.

Within our research, we adopt the idea according to which the term smart city can be used only in the context of information society and its role is twofold: it supports people to perform information exchange and facilitates that exchange by ensuring the information flow infrastructure. We also draw on the European Smart Cities Project report, in an attempt to identify an instrument that would transform citizens into smart people. To start with, an investigation is made to find out if citizens have become aware of the need to have an instrument specific to a smart urban space.

The smart instrument used in this study is a concept map with urban locations, local academic and cultural poles, where events are organised for dissemination of knowledge. In our view, smart people are persons who use technology to simplify their life and assimilate knowledge and are reluctant to technology replacing human decision-making. Such citizens are open to challenges introduced by ICT-s in all areas of life, extend their presence to the virtual space and at the same time maintain their presence in the physical space.

An instrument was designed to measure the physical presence of people in a society increasingly influenced by Web 2.0 technology with the aim of detecting emergent smart features. The social features of $21^{\text {st }}$ century Romanian academic culture are targeted by means of measuring the intensity of individuals' virtual and physical presence during a time interval that pre-dates the smart era.

Our study emphasises that there are people who are able to identify and become aware of the lack of instruments needed to minimise the time for information transmission/exchange; they are also adepts of technology used to simplify their life instead of depending entirely on it. An example of the way smart governance can react to and solve customers' needs is described in the 2010 report on Customer Journey Mapping, during which the manner in which customers are treated during the interaction process attached to various services is recounted. The Journey Mapping report proves to be an extremely useful tool for the description of the customers' experience of services, including thought processes and reactions. Based on this unsupervised learning experience of the studied group, company customer services can be improved, and 
isolated, badly integrated, or duplicated systems can be identified and corrected. In another research study by Lindström, the concept of personas is used as an instrument to identify the requirements and needs of users by collecting data and creating loyalty programmes. According to Cooper et al., in a smart city "a persona is a user model that is represented as a specific set of characteristics for named individuals. These personas are not actual people, but are archetypes: a set of characteristics that have been constructed based on direct observations of the needs and wants of real people." In such studies, a person encapsulates a distinct set of behaviour patterns in relation to a certain product. These patterns are identified by means of interview data and further supported, if the case, by additional quantitative data. The persona concept plays a crucial role in identification of target groups with similar objectives and expectations towards a particular product or service (Lindström). In our study, we will use the concepts of personas and Journey Mapping to measure students' one-to-one interaction to the locations where academic culture events take place and where they are supposed to acquire and develop their academic culture skills. Thus, the paper is written within the frame of smart city oriented towards smart people capable to act "beyond the 'like' button and to take responsibility for the future shape of the city", more particularly university students. It aims to create students' profiles based on their physical and virtual presence, which allows for the computation of the "importance"- "centrality" of the locations-spaces-buildings where they get their academic knowledge and improve their academic skills.

Local interest and preoccupation for the transition to smart cities dates back to 2012-2013, when academics researched and proposed to the private/public sectors the implementation of ICT-s to increase traffic safety, drivers' comfort and increased mobility due to assistive programmes on smart mobile devices (Varga and Nedevschi, Brehar and Nedevschi, Petrovai et al.). Our current research takes the initiative a bit further because it aims to provide an answer as to the readiness of individuals, namely university students, to take a further step towards a smart urban space. The need to map physical presence and information/knowledge collecting flows prompted the creation of a concept map tool. In accordance to the standards of Palmer et al., the instrument can be considered

1. an adequate descriptive model for determining polarisation in smart cities;

2. a prototype exercise of mapping locations and routes destined to interaction with information flows within physical and virtual space. Based on this prototype, a platform can be set to highlight the relevant links between city cultural locations, information/ knowledge and events, suggesting key poles for economic culture promoters;

3. to allow for the identification of the deficiencies of the existing standard initiatives;

4. to clarify the economic data, information and knowledge flows between the major pillars/promoting cultural centres in a large university centre. 


\section{Material and methods}

\subsection{Research Objective and Variables}

The main objective of the study is to investigate if persons with medium to high education are ready to identify the need for an intelligent tool meant to guide them in exploring physical locations to obtain information flows in a short period of time.

The derived objectives of the study are: (1) to create a prototype of the persons who identified the existing need; these are persons whose movement in a physical and virtual space can be measured in a social and professional context; (2) to validate a concept map tool by using a complex combination of statistical methods and data mining, which then allow for application of social network analysis to research patterns of behaviour towards the topics of interest; (3) to validate the findings in the studies by Fortunato, Girvan / Newman, Papadopoulos et al., and Adedoyin-Olowe et al., by investigating if the length of the inter-node pairs can be calculated based on the measurements done in a recommendation system that can be used in hierarchical clustering, more particularly we focus on the network connections shared by two nodes.

\subsection{Data Collection}

Data was collected for a number of 43 senior undergraduate students enrolled in the course of Economic Informatics. Firstly, a brainstorming session was conducted with 10 students to make clear the information needed for the experiment. The answers confirmed the initial research idea, namely that students would be highly interested in concept mapping guidance to reach the locations and events where they acquire academic information-knowledge. The following information was obtained:

1. novice students would appreciate/benefit from having physical locations and routes mapped;

2. the mapping should be done by experts, namely academics and faculty;

3. the available online maps did not offer the necessary information, resulting in waste of time and confusing information;

4. their presence in the virtual world consists of social networking to stay in touch with friends and colleagues, the Moodle platform to access learning materials and virtual libraries subscribed by the university to do their tasks;

5. the average time spent daily to collect specialist information is about 2-3 hours and the average time spent daily on the social networks is about 4-6 hours.

The second step consisted of designing a questionnaire to collect data on the type of information a student can obtain by physical participation to events taking place in the most important academic cultural centres in Cluj. The main objective was to verify whether (1) students in Economics in their final year identify correctly the economic 
academic culture centres and (2) the type of presence (physical versus virtual) student prefer in order to acquire knowledge and develop their academic knowledge. Their answers were expected to configure a concept map of cultural centres of vital importance for students' academic development. The questionnaire format was adopted in view of eliminating the stress factor generated by the unknown, in this case the mapping activity.

Finally, the questionnaire-map was emailed by Facebook to 100 students asked to answer within a day. The response rate was $43 \%$. This procedure was also meant to collect data on the virtual presence of students (on the social networking site) and diminish potential distortion of answers caused by teacher's presence. The evaluation consisted of $[0,1]$ values.

\subsection{The Analysis Tool}

The current research is situated within the framework of empirical research and scientific reflection aimed at finding ways to measure the degree of readiness of smart citizens to correctly adopt the new organization of the real world so that they use technology in their benefit and are not being "used" by technology.

As mentioned earlier, the preliminary data collection was organised as a survey. The questionnaire consisted of two sections: one with demographic data and one for selection of locations as academic culture poles (University, faculties, university and county libraries, etc). For each location, a list of events (training, internships, workshops, etc.) and type of information-knowledge (economic, sociological, psychological and educational, etc) to be acquired were attached. Each person had to choose a value ranging between $[0,1]$. As this was a dichotomous data processing, the statistical method used followed the steps described below:

1. The data statistical analysis was applied to highlight the features of the study participants.

2. A test followed to confirm if the items of the physical presence measuring tool have one dimension, namely one latent factor. This basic rule is known as unidimensionality (Dan) and is connected to another assumption, namely the item local independence (Dan). Consistent with the proposition that a subject's performance for an item can be predicted and explained by the existence of a latent factor or trait, the ensuing relationship between this performance and the latent factors can be described by a monotone increasing function called item response curve or item characteristic curve (Hambleton et al.). This type of analysis estimates the reliability of the instrument by measuring the elements' internal consistency when they correlate well. The reliability was calculated in accordance to van Soom's theory, which states that a measuring 
instrument is perfectly reliable if it has a perfect positive effect $(r=1)$. The interpretation of results was done in compliance with specialist literature (Popa et al.) whereby a scale/test is more reliable when the reliability score is higher than 0.80 (Dan). This was the state when we decided if our instrument has a high degree of reliability/consistency so that the results can be maintained in time and it can be used for similar scenarios in the future.

3. The next step was aimed at finding out the correlating variables. In specialist literature, Hambleton and Jones state that unidimensionality refers to the existence not of only one dimension but of a dominant dimension which can influence test performance, which is called ability or, more generally, latent factor coverage (Dan). In the case of a test measuring presence, we cannot expect the items to cover the pure presence.

4. The principal component analysis (PCA) is conducted to reveal pre-existent structures in a multitude of multivariate data (Culic). These structures are generally expressed by means of variance and covariance of variables and of similarities and dissimilarities between objects. In this sub-stage, we identified the values and vectors of a square matrix obtained by multiplying matrix data (objects) with matrix anti image, a relatively common procedure in matrix calculus. The null hypothesis is that there is no limited set of factors that can determine the presence of citizens in a smart city. The alternate hypothesis is: there is a limited set of factors that can determine the presence of citizens in a smart city. PCA was used to build a decision model based on the answers to the questionnaire/concept map. By correlating these data, a pattern can be formulated to reveal the limited factors that explain the variation of the studied variables. Within this method, the unrepresentative correlations $(<0.3$ from correlation matrix) were eliminated. Other variables were eliminated in the next step based on Kaiser-Meyer-Olkin measurements (the values of the covariation indices from the principal diagonal of the anti image $>0.5$ ).

5. The hierarchical method was applied to determine the number of clusters, followed by MacQueen's k-means improved by E. Diday's dynamic clouds method (Enachescu). The k-means algorithm was used to generate cluster with objects (persons) who share the same behaviour within the research. The hypothesis was: there are differences between the persons' preferences to visit certain locations to get knowledge. We validated the result by applying the hierarchical k-means, which is a top-down hierarchical clustering method using k-means iteratively with $k=2$. This decision was taken in order to (1) determine if similar results can be obtained by using the two methods and (2) determine the existence of potential communities who, related to space, would allow for the idea of graph, in other words would allow us to perform social network analysis. 
6. In order to reach our purpose, we set on to determine a pattern of present persons by means of applying classification algorithms characteristic to the studied context. The Receiver Operating Characteristic (ROC) Regression analysis was used to validate the result given by the classification algorithms (Enachescu). The analysis model completed aimed to outline the profile of active persons. We employed ROC classifier to test the validation of the developed classification model. Up to this phase, we created a statistical analysis oriented towards the individual behaviour that was meant to demonstrate that a community was created based on our instrument. The ROC classifier helped in the measuring of the smart persons' physical presence in space and similar behaviour patterns.

7. In the final part of the analysis, we set out to determine the places that have the highest concentration of smart persons by means of the social network analysis, particularly the calculation of centrality. Centrality is considered the most often used conceptual tool for exploring the actors' (locations) roles within social networks. The centrality degree of a node in an undirected graph is defined as the number of nodes connected to that node. Consistent with (Wasserman, Faust), "central locations have to be more active in the sense that they have the highest number of connections with other actors-locations in the network." According to the same authors, closeness centrality measures the closeness of a node to the other. Consistent with (Freeman), "a node is the sum of geodesics distance from all other nodes, which is defined as the length of the shortest path from one node to another". Consequently, closeness centrality can be used to determine the degree of influence a node has in the network. Another interpretation belongs to (Borgatti), who states that betweenness gives "the share of times that a node $\mathrm{j}$ needs a node $\mathrm{t}$ (whose centrality is being measured) in order to reach j via the shortest path". In this research, we adopted Everett and Borgatti's theory of nodes in a network, according to which (Everett, Borgatti)

- Group Centrality: the centrality degree of a group is the number of actors outside the group that are connected to the member of that group. Different ties to the same actors by different group members are only counted once.

- Group closeness centrality is defined as the normalized inverse sum of distances from the group to all nodes outside the group.

- Group betweenness centrality shows the "proportion of geodesics connecting pairs of non-group members that pass through the group. (Chaoqun et al.)

These three centrality indicators allowed us to measure the role of locations in the personas' participation to certain events in order to obtain knowledge. The results of our research will meet the results of (Chaoqun et al). 


\section{Study Findings}

The present study is conducted along two dimensions, with their respective hypotheses. The first hypotheses for the first dimension are:

$\mathrm{H} 0$. The subjects are not ready to be interpreted as smart persons.

$\mathrm{H} 1$. The subjects are ready to be interpreted as smart persons.

For the second dimension, the hypotheses are as follows:

$\mathrm{HO}$. The behaviour towards the locations does not generate communities that can act as networks. More exactly, by using hierarchical clustering, we will not obtain communities which, related to the physical space, form a graph, namely a network.

$\mathrm{H} 1$. The behaviour towards the locations generates communities that can act as networks. More exactly, by using hierarchical clustering, we will obtain communities which, related to the physical space, form a graph, namely a network.

The descriptive analysis allowed us to create a profile of the participants, with the following characteristics: age: $18-24$ years; urban: $90.7 \%$; rural: $9.7 \%$; gender: $72.1 \%$ females; 27.9 males; concept map knowledge: 46.5 know about it; 53.5 do not know about it.

Next a reliability-validity analysis was performed to obtain an instrument to measure the extent to which students in economics get involved in academic culture life. This was done in a context in which certain activities (for example, learning, information seeking, training, etc) performed traditionally in a physical environment are increasingly replaced by their counterparts in the virtual environment, resulting in a diminished participation to real world events. The instrument measures students' presence in physical locations at events that occur regularly in the life of a person studying economics in Cluj-Napoca and have been traditionally associated to major sources of academic culture. Within this context, the instrument was used to measure if the locations traditionally considered as poles of student life and the associated culture generating events have maintained their place in a society increasingly governed by the virtual dimension. The results of the analysis are:

1. there are differences between the evaluations of the need for students' physical presence in certain location and at events designed for their field of study;

2. the instrument for the analysis of persons' presence in the physical space needs not be reorganised;

3. the instrument items used to measure the physical presence/absence are highly reliable. Following the application of the test, we obtained that the test is sensitive to the measured characteristics: alpha Cronbach $=0.915$. Mean=2.13663 Std.Dv. $=3.29576$, Number of items in scale is 14 , Valid N:344. The alpha value obtained is significant and suggests that the instrument is adequate to the purpose. It follows that 
the test is unidimensional. Within the process of determining the alpha coefficient, the values of the test item discrimination coefficient are determined. They are presented and interpreted as follows: the students (specialists) manifested a moderate to high agreement in appreciation of the presence/absence of the persons to local academic culture life. Average Inter-Item Correlation: 0.697.

The analysis continued with the measuring of the test internal consistency, based on the split-half method. The results are good: Cronbach alpha, full scale: 0.915 Corr. 1st \& 2nd half: 0.948 Split-half reliability: 0.973 Guttman split-half: 0.94 . Notice should be made that test reliability is easily modifiable. Thus, in accordance with reliability coefficient calculation, the number of items is adequate, so the number of questions need not be increased. Yet, if this were the case, the reliability would not be affected significantly. In conclusion, the test has a good reliability and that means that the questions inclined to be a whole, meaning that the halves of the instrument (locations and events) could be used on their own to measure students' physical presence. Because of the alpha Cronbach $=0.915$, it is recommendable that the instrument be used as it was designed. In a parallel test developed with similar elements, students' relative scores would show a slight difference.

Based on the ANOVA test, whose results are presented in the table below, the study hypothesis is unilateral and namely there are differences between items generated by students' answers, meaning that the test can differentiate students in accordance to their physical presence to the respective locations and events.

\begin{tabular}{|l|c|c|c|c|c|}
\hline \multicolumn{7}{|c|}{ Analysis of Variance } \\
\hline & Sums of & Df & Mean & F & p \\
\hline Between Subjects & 266.1204 & 343 & 0.775861 & & \\
\hline Within Subjects & 356.7066 & 4472 & 0.079764 & & \\
\hline Between Items & 62.7137 & 13 & 4.824128 & 73.16769 & 0.00 \\
\hline Residual & 293.9930 & 4459 & 0.065932 & & \\
\hline Total & 622.8270 & 4815 & & & \\
\hline
\end{tabular}

Table 1. Anova test results

The analysis continued with Principal Component Analysis (PCA) by which we tested if our instrument has latent factors, more precisely if there are central and marginal items or they form a whole that can be measured along the same dimension. As known, $\mathrm{PCA}$ provides various results such as descriptive analysis, the correlation matrix, KMO and Bartlett test, communality, total variance, screen plot image, component matrix and rotated component matrix.

The first principal eigenvalue captures $28.93 \%$ of the variability in the data. 


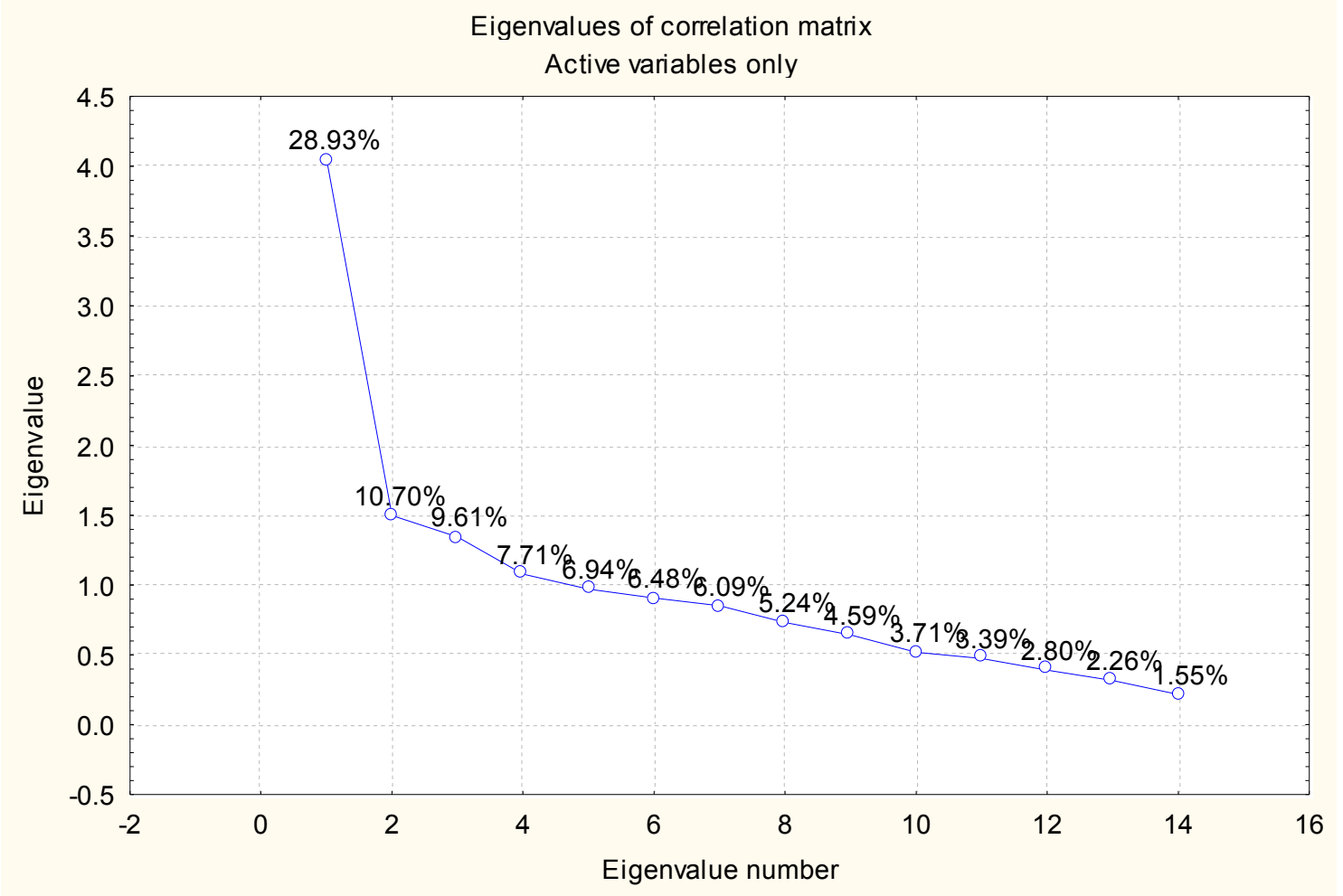

Figure 1. Screen Plot

It is known that coefficients with values higher than 0.6 indicate a close connection between factors and variables. Thus:

1. the first factor may suggest students' interest for self-development and includes the variables: Event CourseLab (0.687); Training (0.569), Workshops (0.405), C1.economic (0.758), C1.linguistic (0.652), C1.psychological and educational (0.550), C1.communication 0.819 .

2. the second factor may suggest students' interest for professional development the variables: C1.internships (0.581); C1.political (0.518), C1.sociological (0.490).

According to communality results and calculation of the square cosines, values are obtained between $[0.268,0.822]$, close to 1 , so we can conclude that the information is well-preserved in the studied physical space.

This method was used to eliminate items with correlations less than 0.3 but such items did not exist. Factor analysis is adequate for the current study, which is a decision taken based on the results of the Kaiser-Meyer-Olkin test (used to measure the extent to which data variation is caused by the instrument). The value of the KaiserMeyer-Olkin test is 0.745 , which shows that factor analysis is indicated in this case. The results of the Bartlett test $p=0.000$ confirm that the analysis is useful on the given data. Consistent with Kaiser's criterion (communality values have to be higher than 
0.4), (Culic) the latent root consists of studied items. Consequently, the null hypothesis is rejected and the alternate is accepted, according to which there is a limited set of items which determine the validity of the instrument in a ratio of $50.76 \%$. Additionally, the second null hypothesis is rejected and the alternative accepted, according to which in the study there are moderately correlated items.

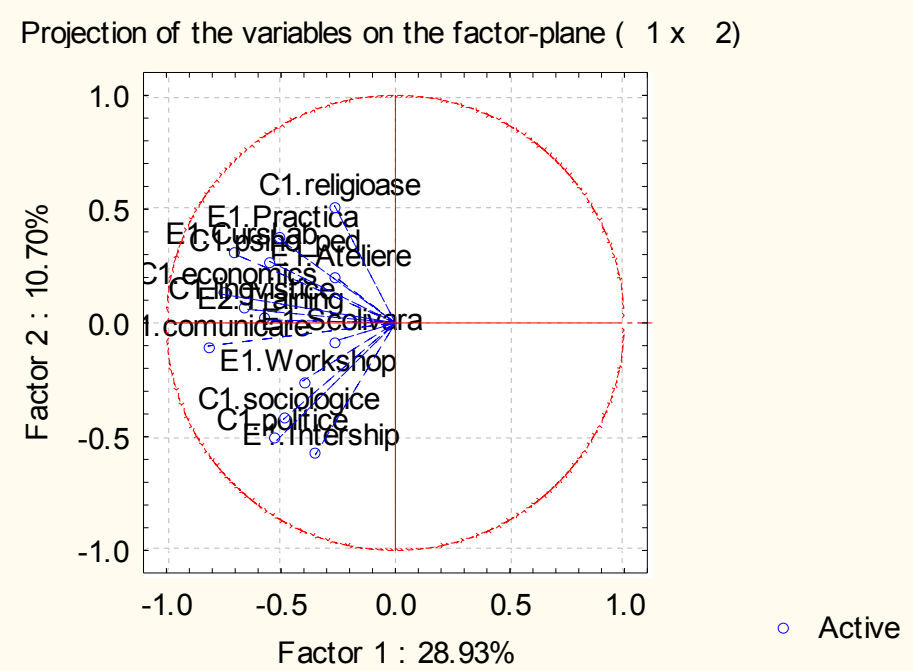

Figure 2. Projection of the variabiles

Next, we applied hierarchical clustering. The hypothesis for this step is that there are differences between personas' evaluation as recognition of the importance of their presence in the physical spaces to obtain information/knowledge. The results of applying the k-means algorithm show that students can be grouped into two clusters characterised by the fact that the value of inter-class inertia is significantly higher than the value of intra-class inertia. The clusters resulted from the way students indicated their presence in the specific locations at the events and for the purpose of certain knowledge transfer activities.

Cluster 1 includes the preferences of students with a low presence and no high fluctuations in their physical presence at academic culture activities intended for selfdevelopment $(\mathrm{F}=65.442 ; \mathrm{p}=0.000)$.

Cluster 2 includes the preferences of students with a high presence and a fluctuating behaviour in activities intended for their professional development $(F=39.869 ; p=0.000)$.

Next, we applied the Manova test to assess the following hypothesis:

$\mathrm{HO}$ : There is no difference between the factors studied for the 2 groups of students distributed according to their participation in the specific locations. 
$\mathrm{H} 1$ : There are differences between the factors studied for the 2 groups of students distributed according to their participation in the specific locations.

As a result of the Manova test, the null hypothesis was rejected and the alternative hypothesis was accepted: Wilks' Lambda $(F=1.512, p=0.000)$. Cluster analysis produced student clusters which show inherently similar behaviour patterns. The students with good physical presence are grouped in a cluster, being considered a main reference in the process of improving the characteristics of the other students in the same cluster. The graph of the two clusters is presented below:

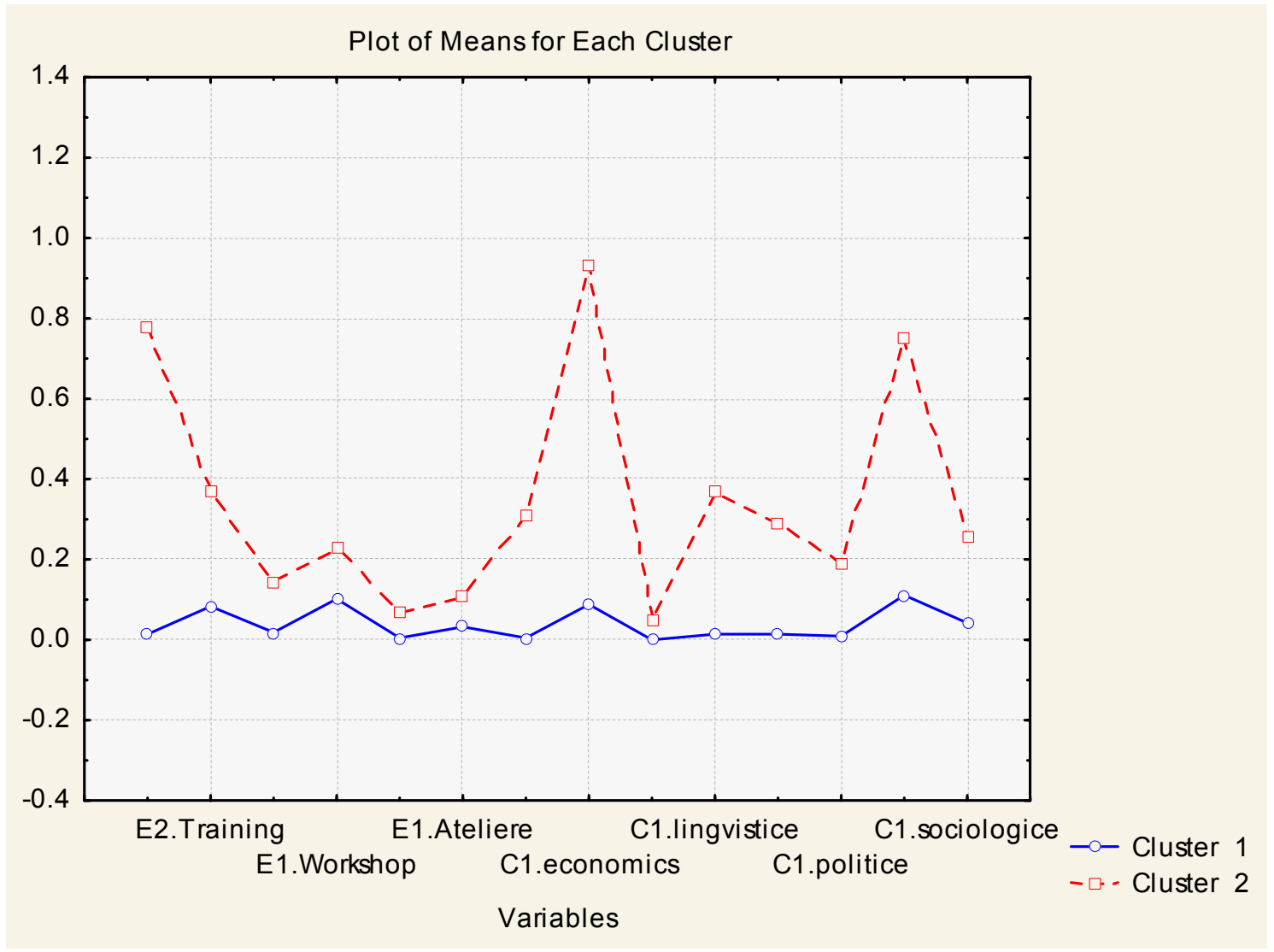

Figure 3. Physical presence clusters

The study continued by applying ROC curve to find out if the studied attributes are predictive for the model of determining the students' physical presence in the academic culture life of the city.

The area below the curve is $0.986, p=0.001 ; 95 \% \mathrm{Cl}(0.978 ; 0.999)$. This is a discriminating model, eliminating the false negatives and the false positives for $92 \%$ of the cases. 


\section{ROC Curve}

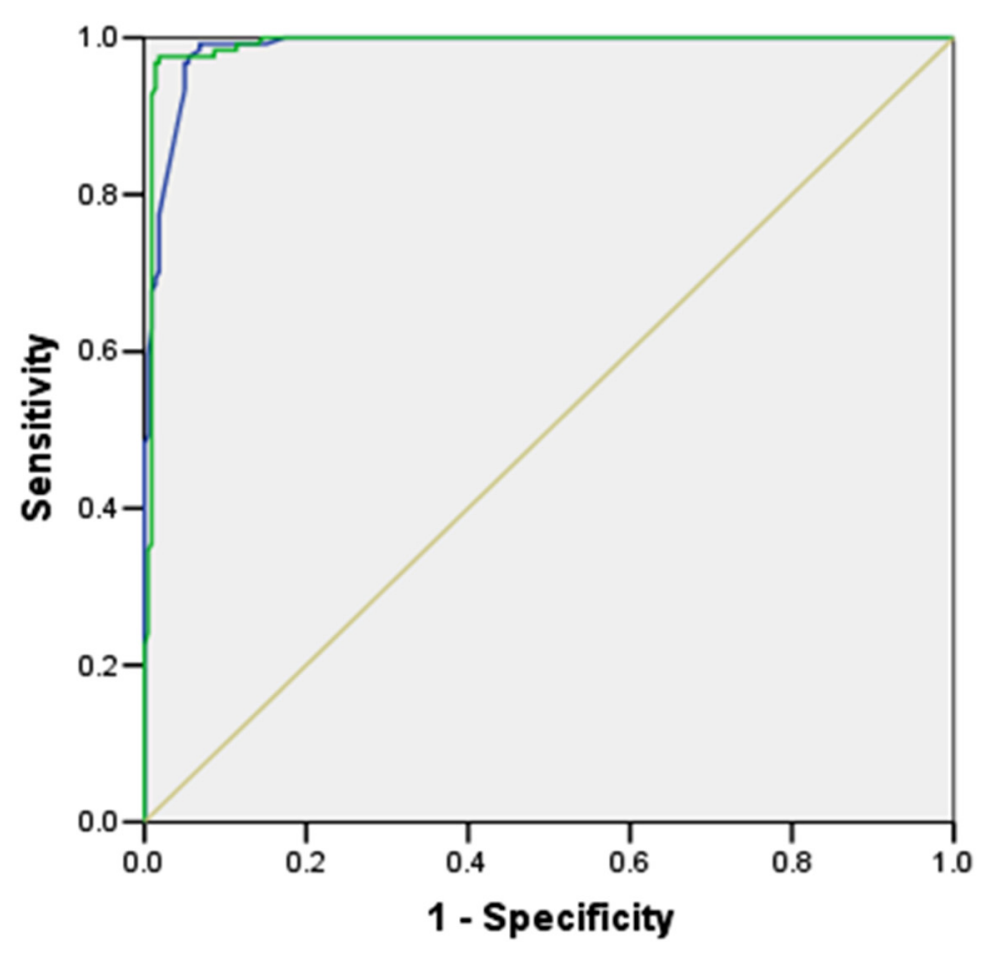

Source of the Curve - Logistic Pred Prob -Discriminant Pred Prob Reference Line

Diagonal segments are produced by ties.

Figure 4. ROC Curve

Thus, the null hypothesis of the study is rejected and the alternate hypothesis is accepted, according to which the subjects under study are ready to be interpreted as smart persons.

Moreover, two behaviour clusters were obtained, which may indicate that these persons can also be interpreted as same behaviour communities, representing a graph. Consequently, social network analysis can be applied to determine what the most important locations are, namely the poles where knowledge transfer is made by means of events.

In order to establish the presence/absence of the poles, we set out to determine the values of the graph nodes to obtain a classification in accordance with the frequency of use of the respective locations. In the specialist literature, there are two approaches to measuring the "importance" of a node: - the importance of the nodes is established in accordance to the importance level of the information flow that passes through the nodes (Bonacich); - the nodes represent a concentration of information groups with the corollary that the network is an image of the data groupings based on features studied in the research (Borgatti). 
Our approach draws on the second author (Borgatti). This means that we focussed on the term "importance" with the aim of determining the role presence and localisation have in information-knowledge transfer. In order to carry out this stage, we used the classical topological measuring methods, more specifically we calculated the degree of centrality (Nieminen), (Freeman), the closeness centrality (Freeman), (Sabidussi), (Wasserman, Faust), the betweenness centrality (Freeman) and the eigenvector centrality (Latora, Marchiori). The theoretical background for the indicators is presented in detail in (Nieminen), (Freeman), (Sabidussi), (Carrington, Scott, Wasserman) and (Latora, Marchiori). Based on these indicators (Cadini, Petrescu), data were processed to obtain a topological network of importance.

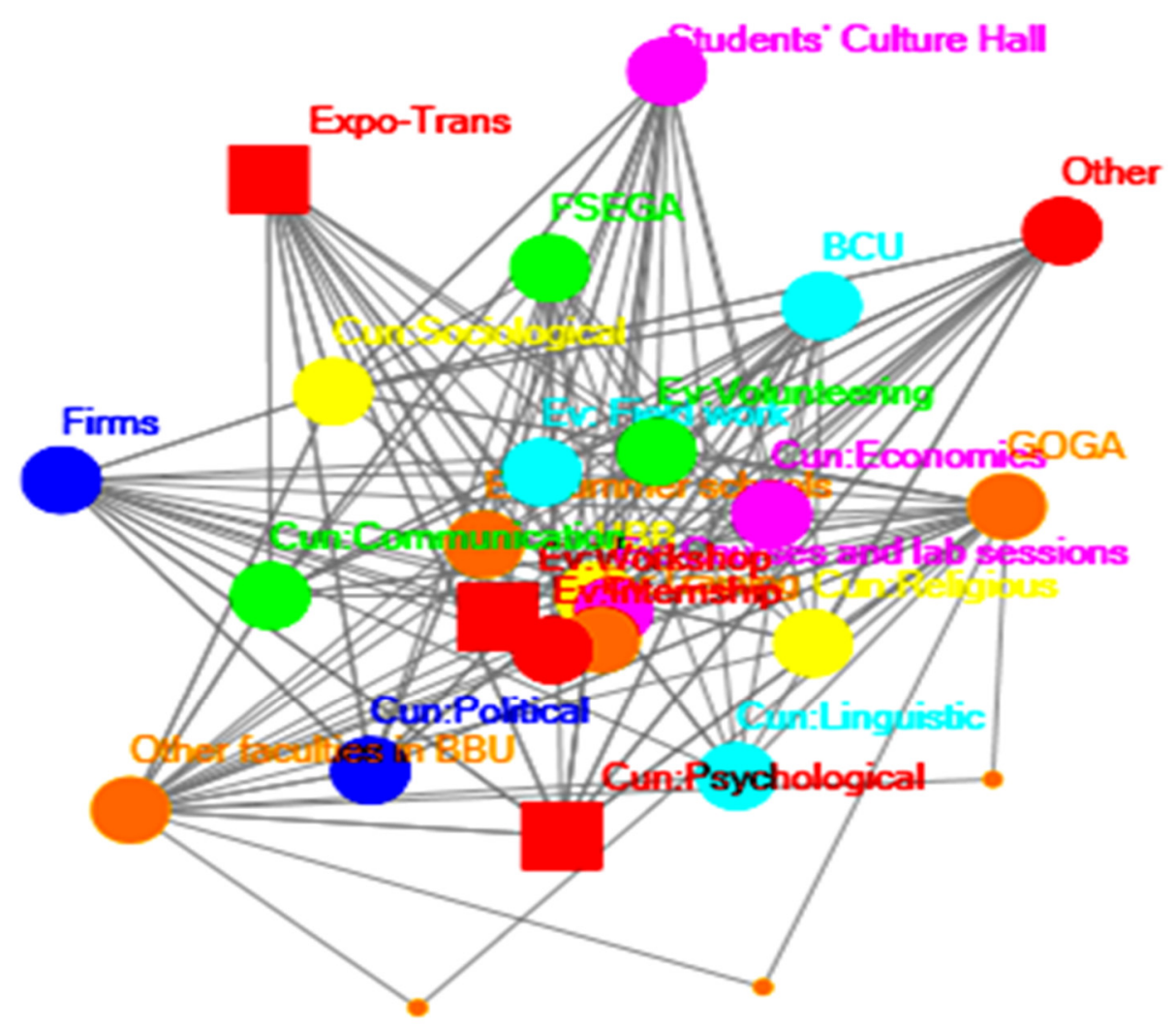

Figure 5. Closeness Centrality of location

Each "edge" represents a link between two locations and events, respectively information/knowledge acquired.

The edges represent different types of connections that can be created during the information/knowledge transfer occurring at those specific places. A "tie" edge is created in the moment when a user visits the location to be subject to information/ knowledge transfer during an event. 


\begin{tabular}{|l|l|l|l|l|}
\hline Vertex & Degree & $\begin{array}{l}\text { Betweenness } \\
\text { Centrality }\end{array}$ & $\begin{array}{l}\text { Closeness } \\
\text { Centrality }\end{array}$ & $\begin{array}{l}\text { Eigenvector } \\
\text { Centrality }\end{array}$ \\
\hline BBU & 21 & 1.000 & 1.276 & 0.285 \\
\hline Ev: Courses and lab sessions & 6 & 0.027 & 1.897 & 0.084 \\
\hline Ev:Training & 6 & 0.027 & 1.897 & 0.084 \\
\hline Ev:Internship & 9 & 0.081 & 1.690 & 0.123 \\
\hline Ev:Workshop & 9 & 0.081 & 1.690 & 0.123 \\
\hline Ev:Summer schools & 9 & 0.081 & 1.690 & 0.123 \\
\hline Ev: Field work & 9 & 0.081 & 1.690 & 0.123 \\
\hline Ev:Volunteering & 9 & 0.081 & 1.690 & 0.123 \\
\hline Know:Economics & 9 & 0.081 & 1.690 & 0.123 \\
\hline Know:Religious & 9 & 0.081 & 1.690 & 0.123 \\
\hline Know:Linguistic & 9 & 0.081 & 1.690 & 0.123 \\
\hline Know:Psychological & 9 & 0.081 & 1.690 & 0.123 \\
\hline Know:Political & 9 & 0.081 & 1.690 & 0.123 \\
\hline Know:Communication & 9 & 0.081 & 1.690 & 0.123 \\
\hline Know:Sociological & 9 & 0.081 & 1.690 & 0.123 \\
\hline$\ldots \ldots .$. & $\ldots .$. & $\ldots$ & $\ldots$ & $\ldots$ \\
\hline
\end{tabular}

Table 2: Topological and Reliability Centrality Measures

Centrality is considered one of the most important and frequently used conceptual tools for assigning roles to the subjects studied in social networks. In the current study, between's centrality is approached as measure of the degree of connection between locations in a network for information-knowledge transfer in physical space. The findings indicate that the studied locations act as ties between events and information-knowledge.

Finally, based on the centrality measuring, we explored the place of each location in relation to information-knowledge transfer within specific events. The values Graph Density - 0.42, Maximum Geodesic Distance (Diameter) - 3, Average Geodesic Distance 1.59 , show that not all nodes are interconnected. The basic structure is non-hierarchical, within which all locations play an equal role in information-knowledge transfer taking place during specific events. The resulting network facilitates common comprehension, a knowledge centre and support for students' information-knowledge acquisition.

\section{Discussion}

The current paper proposes a prototype tool for measuring the presence of individuals in the physical and virtual space. The objective was to look into the theory according to which in smart cities, people's physical presence will diminish and be 
replaced by a 'like'. The participants to the study were senior undergraduate students of Economic Informatics. The need for a concept map was validated, then a survey based on its elements collected the data. The main null hypotheses of the study were rejected and the alternate are accepted. According to these:

1. the participants in the study are ready to be interpreted as smart persons, more exactly as persons who use technology to simplify their life and to obtain an adequate information flow in a short time.

2. Behaviour towards the specific locations generates communities which in relation to the physical space create a graph, a network and, by extrapolation, a social network. The latter can be analysed using specific methods to determine poles that attract concentrations of persons. Consequently, problems in which the variables studied in relation to space can be demonstrated to generate graphs may be solved based on data mining and social network analysis so as to obtain more rigorous results.

Consequently, the profile of the smart person obtained can be argued to deny the theory according to which urban ICT-s may diminish people's physical presence in favour of virtual presence measured by 'like'-s. In our view, smart persons are much more focussed on using both sides of reality to achieve their purpose.

3. The results of the final part of our research validates the findings of(Freeman), (Stanca and Felea), (Palmer, Alfi and Dam). Two clusters were obtained that could be interpreted as communities sharing the same behaviour. By their physical presence at the specific locations, the members of the two communities can be represented as in (Adedoyin-Olowe, Gaber and Stahl) by a graph. The hierarchical clustering methods helped us to identify the poles of academic culture by the number of persons visiting them to acquire information-knowledge. These poles were validated in relation to real physical space. Thus, findings indicate that the length of the inter-node pairs can be calculated based on the measurements done in a recommendation system that can be used in hierarchical clustering, more particularly by focussing on the network connections shared by two nodes.

The intermediate results that lead to major results are:

1. The concept map is a valid tool and can be used to guide students through the most important routes for extracting academic information-knowledge. It is also a useful tool to reduce the time for offering and finding information. If generalised at the level of student population, it could also be used for obtaining information about the student interactions with the physical environment or about their routes.

2. The findings of multivariate analysis suggest that the proximity of association between locations, events and type of information-knowledge transfer reveal that the respective individuals have chosen to participate socially to the economic space 
created. Values also suggest that students are still adepts of physical participation to the events organised with the aim of economic information-knowledge transfer within academic environment.

3. The study suggests that locations are visited/chosen as a consequence of the type of events and information-knowledge transfer taking place; this may suggest students' interest in the contents of the events and probable benefit for their self and professional development.

4. The findings confirm that there is a significant group of students who have intense physical presence but there is also a group who show increased virtual presence and diminished physical presence.

\section{Conclusions}

The study demonstrates that, in order to become a smart city, an important East-European city needs people who are ready to undertake the transition but tools need to be devised to measure readiness so as to avoid issues caused by cultural shock. The current findings indicate that 18 to 26 year old people (students) still need to be physically present in locations where information-knowledge transfer is performed. Moreover, in spite of being in their final undergraduate year, these students still need guidance to define this physical presence for their personal and professional development. On the other hand, students' virtual presence has been noted on social networks and e-learning platforms. However, the current study confirms the findings of previous research (started in 2009) where students that use ICT-s as a subsidiary were noticed to use learning platforms only under constraint. Currently, though, mature students knowledgeable in economics and ICT-s are aware of technology utility and it is our opinion that citizens should be supported in the process of transition so as a too abrupt a change to a smart city should not generate passive citizens adepts of "like"-s.

Future research will extend the study to junior undergraduates in economic studies to examine if the education level plays a role in the transformation in smart people. Experiments could be approached from the angle of dynamic decisions theory to determine if learning the environment has an effect on completing a route. In practical terms, the authors intend to design a virtual application to assist the students to discover the cultural space in Cluj so that they may extract the informationknowledge they need in the shortest time possible.

\section{Works Cited}

A. Androniceanu, M. Ivan. "Smart City” - A Challenge For The Development Of The Cooperation Mechanism Between European Cities." Proceedings of Administration and Public Management International Conference, Issue: 8 (2012), pp. 333-346. 
M. Adedoyin-Olowe, M. M. Gaber, F. Stahl. "A Survey of Data Mining Techniques for Social Network Analysis." jdmdh.episciences.org/18.pdf, last accessed 2014.

P. Bonacich. "Power and Centrality: A Family of Measures". American Journal of Sociology (University of Chicago Press) 92:(1987), pp.1170-1182

S. P. Borgatti. "Centrality and network flow." Social Networks, 27(1) (2005), pp. 55-71.

R. Brehar, S. Nedevschi. "Scan Window Based Pedestrian Recognition Methods Improvement by Search Space and Scale Reduction." Proceedings Intelligent Vehicles Symposium, Dearborn, USA, 2014, pp. 529-534.

F. Cadini, E. Zio, C-A. Petrescu. "Using centrality measures to rank the importance of the components of a complex network infrastructure." Critical information infrastructure security, Springer Berlin Heidelberg, 2009, pp. 155-167.

P. J. Carrington, J. Scott, S. Wasserman. "Models and Methods in Social Network Analysis." New York: Cambridge University Press, 2005

A. Cooper, R. Reimann, D. Cronin. "About Face 3: The Essentials of Interaction Design." John Wiley \& Sons, 2007.

I. Culic. "Metode avansate în cercetarea social - Analiza multivariată de interdependență." Editura Polirom, 2004.

C. O. Dan. "Caracteristici ale modelelor de evaluare psihologică bazate pe teoria răspunsului la Item." www.academia.edu/5218682/Characteristics_of_ psychological_assessment_models_based_of_Item_Response_Theory_Rom anian, 2012, last accessed 2014.

D. Enachescu. "Data maining, Metode și aplicații." Editura Academiei Române, București, 2009.

M. Everett, S. P. Borgatti. "Extending centrality, Models and Methods in Social Network Analysis." Cambridge University Press, Cambridge, UK, 2005, pp. 57-76.

C. Felea, L. Stanca. "Wikis in Teaching English for Academic Purposes - Engaging Romanian Students in a $21^{\text {st }}$ Century Learning Environment. eLSE, elearning and Software for Education." April 28-29, Bucharest. Editura Universitară, (2011) 460-467.

C. Felea, L. Stanca. "Wiki Tools in Teaching English for Academic Purposes - An Analysis of Student Learning Behaviour Pattern." "CAROL I" National Defence University Publishing House. Vol. 1, (2013) 82-91.

C. Felea, L. Stanca. "Wiki Tools in Teaching English for Academic Purposes - IT and Language Proficiency as Predictors of Online Participation." "CAROL I" National Defence University Publishing House, Vol 3, (2013) 312-321.

C. Felea, L. Stanca. "Wiki Tools and English for Academic Purposes-Fostering Collaborative and Autonomous Learning in Higher Education. Revista de Informatică Socială, No. 14, (2010) 55-65. 
S. Fortunato. "Community detection in graphs." Physics Reports 486(3) (2010), pp. $75-174$.

L.C. Freeman. "Centrality in social networks conceptual clarification." Social Networks, 1(3) (1979), pp. 215-239.

M. Girvan, M.E. Newman. "Community structure in social and biological networks." Proceedings of the National Academy of Sciences, 99(12) 2002, pp. 7821-7826.

R. Giffinger, C. Fertner, H. Kramar, R. Kalasek, N. Pichler-Milanovic, E. Meijers. "Smart Cities Final Report." Vienne: Centre of Regional Science. Retrieved 12 May, 2012, from: www.smart-cities.eu/download/smart_cities_final _report.pdf, last accessed 2014.

R. Hambleton, H. Swaminathan, J.H. Rogers. "Fundamentals of Item Response Theory." London: Sage Publications Inc. 1991.

R. K. Hambleton, R. W., Jones. "Comparison of classical test theory and item response theory and their applications to test development." Educational Measurement: Issues and Practice, 12(3) (1993), pp. 38-47.

R. G. Hollands. "Will the real smart city please stand up?" City, Taylor \& Francis Journals, vol. 12(3) (2008), pp. 303-320.

N. Kominos. "Intelligent cities: innovation, knowledge, systems and digital spaces." London. Spon Press. 2002.

A. Ionita, R. Ilie. "Conceptul de Oras On-Line." on-line: rria.ici.ro/ria2000_2 /art05.html, last accessed 2014.

S. Wasserman, K. Faust. "Social network analysis: Methods and applications." New York: Cambridge University Press, 1994.

V. Latora, M. Marchiori, "A Measure of Centrality Based on the Network Efficiency", New Journal of Physics 9, 2007,pp.188-197

M. Lindström. "Report-Making customer group real-using Personas." Smart Cities Brief, No.10, 31 January 2011, www.smartcities.info/files/365_42759_Smart_ Cities_Research_Brief_Using_Personas.pdf accessed November 25, 2014. last accessed 2014

P. Lombardi. "New challenges in the evaluation of Smart Cities." Multi-sector, Network Industries Quarterly, vol. 13, no. 3, 2011, http://newsletter.epfl.ch/mir/ index. php? module $=$ epflfiles\&func $=$ getFile\&fid $=241$ \&inline=1, last accessed 2014 .

J. Nieminen, "On Centrality in a Graph. Scandinavian", Journal of Psychology, n.15, 1974, pp. 322-336.

Chaoqun Ni, C. R. Sugimoto, J. Jiang. "Degree, Closeness, and Betweenness: Application of group centrality measurements to explore macro-disciplinary evolution diachronically."

elektrokomponenten.ch/media/files/09e4150bd20e58c913000000.pdf, last accessed 2014. 
D. Palmer, S. Alfi, K. van Dam. "Mapping Smart City Standards- Based on a data flow model." www.bsigroup.com/LocalFiles/en-GB/smart-cities/resources/BSI-smart-cities-report-Mapping-Smart-City-Standards-UK-EN.pdf, last accessed 2014.

S. Papadopoulos, Y. Kompatsiaris, A. Vakali, P. Spyridonos. "Community detection in social media." Data Mining and Knowledge Discovery. 24(3) (2012), pp. 515554.

K. Paskaleva. "Enabling the smart city: The progress of e-city governance in Europe." International Journal of Innovation and Regional Development. No. 1 (2009), pp. 405-422.

A. Petrovai, A. Costea, F. Oniga, S. Nedevschi. "Obstacle detection using stereovision for Android-based mobile devices." IEEE International Conference on Intelligent Computer Communication and Processing, Romania, (2014), pp. 141-148.

N. Popa, L. Antonesei, A. V. Labăr. "Ghid pentru cercetarea educației, Editura PoliromCollegium, 2009.

G. Sabidussi. "The centrality index of a graph." Psychometrika 31: (1966), pp. 581-603.

J. M. Shapiro. "Smart cities: quality of life, productivity, and the growth effects of human capital." The Review of Economics and Statistics, 88 (2) (2008), pp. 324-335.

E. van Soom. "Measuring levels of supply and demand for e-services and e-government: a toolkit for cities." Smart Cities Research Brief, N. 3:2009.

<http://www.smartcities.info/files/Smart_Cities_Research_Brief_Measuring_ levels_of_supply_and_demand_for_eservices_and_egov.pdf >, accessed November 25, 2014, last accessed 2014.

C. Spearman. "General Intelligence." American Journal of Psychology.15 (1904), pp. 201-293.

R. Varga, S. Nedevschi. "Label transfer by Measuring Compactness." IEEE Transactions on Image Processing, Vol.22, 2013, pp. 201-210.

Report, "Customer Journey Mapping." Smart Cities Brief, No.12, 2010 http://www.smartcities.info/files/Smart_Cities_Brief_Guide_to_Customer_ Journey_Mapping.pdf>, last accessed 2014. 